\title{
Representation of Planar Hypergraphs by Contacts of Triangles
}

\author{
Hubert de Fraysseix, Patrice Ossona de Mendez, and Pierre Rosenstiehl \\ CAMS UMR 8557, CNRS/EHESS, Paris, France \\ $\{$ hf , pom, pr $\}$ @ehess.fr
}

\begin{abstract}
Many representation theorems extend from planar graphs to planar hypergraphs. The authors proved in 10 that every planar graph has a representation by contact of triangles. We prove here that this representation result extend to planar linear hypergraphs. Although the graph proof was simple and led to a linear time drawing algorithm, the extension for hypergraphs needs more work. The proof we give here relies on a combinatorial characterization of those hypergraphs which are representable by contact of segments in the plane, We propose some possible generalization directions and open problems, related to the order dimension of the incidence posets of hypergraphs.
\end{abstract}

\section{Introduction}

\subsection{Hypergraphs}

A hypergraph $\mathcal{H}$ is an ordered pair $(X, \mathcal{E})$ where $X$ is a finite set whose elements are called vertices and $\mathcal{E}$ is a collection of nonempty subsets $E$ of $X$ called edges whose union is $X$. Two vertices $x, y \in X$ (resp. two edges $E, E^{\prime} \in \mathcal{E}$ ) are adjacent if $\{x, y\}$ is included in some edge of $\mathcal{H}$ (resp. if $E \cap E^{\prime}$ is not empty). A hypergraph is linear if any two edges have at most one common element: $\forall E, E^{\prime} \in \mathcal{E}:\left|E \cap E^{\prime}\right| \leq 1$. A loop of a hypergraph is an edge of cardinality 1. A loopless linear hypergraph is said to be simple. We note $n(\mathcal{H})=|X|$ the order of $\mathcal{H}$ and $m(\mathcal{H})=|\mathcal{E}|$ its size. The sub-hypergraph of $\mathcal{H}$ induced by a subset $A \subseteq X$ is the hypergraph $\mathcal{H}_{A}=\left(A, \mathcal{E}_{A}\right)$, where $\mathcal{E}_{A}=\{E \cap A: \quad E \in \mathcal{E} ; E \cap A \neq \emptyset\}$. The representative graph $\mathrm{B}(\mathcal{H})$ of $\mathcal{H}$ is the black and white colored bipartite graph whose set of white vertices is $X$, whose set of black vertices is $\mathcal{E}$ and whose edges are those pairs $\{x, E\}$ such that $x \in E \in \mathcal{E}$. For terms in Hypergraph Theory, not specifically defined here, we refer the reader to [2].

Different generalizations of the concept of graph planarity to hypergraphs have been considered (See [13], for instance). Zykov proposed to represent the edges of a hypergraph by a subset of the faces of a planar map 28. Walsh has shown that Zykov's definition (as well as another definition by Cori [4]) is equivalent to the following: A hypergraph is planar if and only if its representative graph $\mathrm{B}(\mathcal{H})$ is planar [26] (See also [5 14 27]). In the figures, hypergraphs are displayed by mean of their representative graph. 


\subsection{Geometrical Representations}

Geometrical representations of planar graphs gained much attention these last three decades, particularly since planar graphs have been proved to have a straight line representation on a linear size grid [11|12|23. Other geometrical representations have been proposed, like rectilinear representations (a.k.a. visibility representations) [19|24], representations by contacts of convex sets (for instance, circles [1]15] or triangles [10]) and representations by contacts or intersections of Jordan arcs or of straight line segments [7/9|8|21].

Contacts systems of segments are studied in 918 . For instance, it is proved there that a graph is the contact graph 11 of a family of segments if and only if any subgraph induced by a subset of $p \geq 2$ vertices has at most $2 p-3$ edges. In particular any triangle-free planar graph is the contact graph of a family of segments (and this result extends to "triangle-free" planar hypergraphs). Not every planar graph (thus not every planar linear hypergraph) is representable by contacts of segments. The characterization of those planar linear hypergraphs which are contact hypergraphs of segments and points is recalled and discussed in Section 2 ,

A possible relaxation stands in allowing intersections. In [20, Scheinerman asked whether any planar graph could be represented as the intersection graph of a family of segments. Chalopin, Gonçalves and Ochem recently claimed to have proved this conjecture. The authors have proposed the following strengthening of Scheinermann's conjecture.

Conjecture 1. Any planar linear hypergraph is the intersection hypergraph of a family of segments.

Another possible relaxation stands in considering triangles instead of straight line segments. Actually, it is well known that any planar graph is the contact graph of a family of convex sets in the plane. Different kinds of convex sets have been considered, like circular disks 1115. However, as no three circular disks can be in contact at a common point, this representation fails to extend to hypergraphs. In [10, it is proved that any planar graph can be represented as the contact graph of a family of triangles in the plane. The aim of this paper is to extend this later result to planar linear hypergraphs.

\subsection{Further Definitions on Graphs}

For a subset $X$ of vertices of a plane graph $G$, we denote $\mathcal{N}(X)$ the union of $X$ and the set of the neighbors of the vertices in $X$ in $G$, by $G[X]$ the subgraph of $G$ induced by $X$. The set of edges incident to a vertex in $X$ and a vertex out of $X$ is denoted by $\omega(X)$. When $G$ is directed, $\omega^{-}(X)$ denotes the set of the edges of $\omega(X)$ which are oriented toward $X$.

We denote by $\operatorname{Extr}(\mathrm{X})$ the vertex set of the outer face of $G[X]$. The Closure of $X$ is the union of all the subsets $Y$ such that $\operatorname{Extr}(Y)=\operatorname{Extr}(X)$. Notice that

\footnotetext{
${ }^{1}$ To be precise, we only allow one-sided contacts, see Section 2 for more details.
} 
$\operatorname{Clos}(X)=\operatorname{Clos}(Y)$ if and only if $\operatorname{Extr}(X) \subseteq Y \subseteq \operatorname{Clos}(X)$. The subset $X$ is closed if $X=\operatorname{Clos}(X)$. The subset $X$ of vertices is a disk of $G$ if $X$ is closed, has cardinality at least 3 , and the outer face of $G[X]$ is a cycle.

\section{Contacts of Segments or Pseudo-segments}

A finite set of Jordan arcs is called a family of pseudo-segments if every pair of arcs in the set intersects in at most one point. In particular, any family of segments is a family of pseudo-segments. A one-sided contact family of pseudosegments and points is a couple $(\mathcal{A}, P)$, where $\mathcal{A}$ is a family of pseudo-segments that may touch (only on one side at each contact point) but may not cross and whose union is connected, and where $P$ is a set of points in the union of the pseudo-segments including all the extremities of the pseudo-segments. For the sake of simplicity we shall use the term of contact system instead of "one-sided contact family" in the remaining of the paper.

Each contact system $(\mathcal{A}, P)$ defines a connected bipartite plane graph $G=$ $\left(V_{\circ}, V_{\bullet}, E\right)$, its incidence graph, where $V_{\circ}$ corresponds to the pseudo-segment set, $V_{\bullet}$ corresponds to the point set and $E$ corresponds to the set of incidences between points and pseudo-segments.

The graph $G$ has no cycle of length 4 (as two pseudo-segments share at most one point) thus has girth at least 6 . The planar linear hypergraph $\mathcal{H}$ whose representative graph is $G$ is the contact hypergraph of $(\mathcal{A}, P)$. When no three pseudo-segments of $\mathcal{A}$ touch at a single point, the contact hypergraph actually is a graph, which we shall call the contact graph of $(\mathcal{A}, P)$. Contact hypergraphs of pseudo-segments have been characterized in [7] and contact hypergraphs of segments have been characterized in [8] and [9].

Moreover, the contact system also defines an orientation of $G$ : if $x \in V$ • corresponds to a point $p$ on a pseudo-segment $S$ corresponding to $y \in V_{\text {o }}$, the edge $\{x, y\}$ is oriented from $x$ to $y$ if $p$ is an extremity of $S$ and from $y$ to $x$, otherwise. This orientation is such that the indegree of a vertex in $V_{\circ}$ is exactly 2 and the indegree of a vertex in $V \bullet$ is at most 1 . We call such an orientation a $(2, \leq 1)$-orientation. The converse is quite simple to prove (see [7]):

Theorem 1. A directed bipartite plane graph $G=\left(V_{\circ}, V_{\bullet}, E\right)$ is the incidence graph of a contact system of pseudo-segments and points with the embedding and the orientation induced by the contact system if and only if $G$ has girth at least 6 , and the orientation of $G$ is a $(2, \leq 1)$-orientation.

The characterization of incidence graphs of segments and points given in [8 (Theorem 38) can be stated in a similar way:

Theorem 2. A directed bipartite plane graph $G=\left(V_{\circ}, V_{\bullet}, E\right)$ is the incidence graph of a contact system of segments and points with the embedding and the orientation induced by the contact system if and only if $G$ has girth at least 6 , the orientation of $G$ is a $(2, \leq 1)$-orientation and each subset $A \subseteq V_{\circ}$ which has cardinality at least two is such that $G[\mathcal{N}(A)]$ has at least three sources on its outer face. 
To consider disks instead of neighborhoods in the last condition, we introduce a new function. For a subset $X$ of vertices, $\sigma(X)$ denotes be the sum of $\omega^{-}(\operatorname{Clos}(X))$ and the number of sources of $G$ in $\operatorname{Extr}(X)$.

Lemma 1. Let $G=\left(V_{\circ}, V_{\bullet}, E\right)$ be a directed bipartite plane graph with girth at least 6 , whose orientation is a $(2, \leq 1)$-orientation.

Then $G$ is the incidence graph of a contact system of segments and points with the embedding and the orientation induced by the contact system if and only if $\sigma(D) \geq 3$ for every disk $D$ of $G$.

Proof. Assume $G$ is the incidence graph of a contact system of segments and points with the embedding and the orientation induced by the contact system and let $D$ be a disk of $G$. It is easily checked that $\sigma(D)$ is the number of sources of $G\left[\mathcal{N}\left(D \cap V_{\circ}\right)\right]$ lying on its outer face. Thus $\sigma(D) \geq 3$ according to Theorem 2

Conversely, assume $\sigma(D) \geq 3$ for every disk $D$ of $G$ and let $A \subseteq V_{\circ}$ be a subset of a least two white vertices of $G$. It is easily checked that the number of sources of $G[\mathcal{N}(A)]$ lying on its outer face is equal to $\sigma(\mathcal{N}(A))$. As $\sigma(X)=\sigma(\operatorname{Clos}(X))$ and as $G$ has girth at least 6 , the inequality $\sigma(\mathcal{N}(A)) \geq 3$ will follow from the statement that $\sigma(X) \geq 3$ for every closed subset $X$ such that $\left|\operatorname{Extr}(X) \cap V_{\circ}\right| \geq$ 2, that we shall prove by induction on $\left|\operatorname{Extr}(X) \cap V_{\circ}\right|$.

If $\left|\operatorname{Extr}(X) \cap V_{\circ}\right|=2$ then $G[X]$ includes no cycle, thus $\left|\operatorname{Extr}(X) \cap V_{\circ}\right|=2$ and $\sigma(X) \geq 3$, by an easy case analysis. Assume $\sigma(X) \geq 3$ for every closed subset $X$ such that $2 \leq\left|\operatorname{Extr}(X) \cap V_{\circ}\right| \leq k$ and let $X$ be a closed subset with $(k+1) \geq 3$ white vertices. If $G[X]$ is disconnected, then either one connected component $G\left[X^{\prime}\right]$ of $G[X]$ has at least two white vertices on its outer face and the result follows from $\sigma(X) \geq \sigma\left(X^{\prime}\right)$ and the induction, or $G$ has at least one connected component $G\left[X^{\prime}\right]$ with exactly one white vertex on its outer face, and the result follows from $\sigma(X) \geq \sigma\left(X \backslash X^{\prime}\right)$ and the induction. If $G[X]$ has a vertex $v$ of degree 1 on its outer face, it is easily checked that $\sigma(X) \geq \sigma(X-v)$ thus $\sigma(X) \geq 3$ according to the induction. Otherwise, if the outer face of $G[X]$ is a cycle, then $X$ is a disk and $\sigma(X) \geq 3$ by assumption. Otherwise, there exists closed subsets $X_{1}, X_{2}$ such that $\left|X_{1} \cap X_{2}\right|=1$ and $X=X_{1} \cup X_{2}$ and each of $G\left[X_{1}\right]$ and $G\left[X_{2}\right]$ includes at least a cycle (thus includes at least two white vertices on its outer face). By induction, $\sigma\left(X_{1}\right) \geq 3$ and $\sigma\left(X_{2}\right) \geq 3$. As it is easily check that $\sigma(X) \geq \sigma\left(X_{1}\right)+\sigma\left(X_{2}\right)-2$, we get $\sigma(X) \geq 4$.

\section{Contacts of Triangles and Segments}

Main Theorem. Any planar linear hypergraph is the contact hypergraph of a family of triangles and segments.

We shall first state some preliminary lemmas and explicit a few transformations on bipartite plane graphs. First recall the following orientation lemma, whose proof has been included for the sake of completeness.

Lemma 2 ([16]). Let $G$ be a multigraph, let $\lambda$ be a mapping from $V(G)$ to $\mathbb{N}$. Then there exists an orientation of $G$ such that each vertex $v \in V(G)$ has indegree bounded by $\lambda(v)$ if and only if 


$$
\forall A \subseteq V(G): \quad|E(G[A])| \leq \sum_{v \in A} \lambda(v) .
$$

Moreover, this orientation is such that each vertex $v$ has indegree $\lambda(v)$ if and only if we also have the global condition $|E(G)|=\sum_{v \in V(G)} \lambda(v)$.

Proof. If every vertex $v$ has indegree bounded by $\lambda(v)$ then the number of edges of $G[A]$ is bounded by $\sum_{v \in A} \lambda(v)$ for any subset $A$ of vertices.

Conversely, assume (10) holds. To any orientation $\mathcal{O}$ associates the non negative integer value $f(\mathcal{O})=\sum_{v: \mathrm{d}^{-}(v)>\lambda(v)}\left(\mathrm{d}^{-}(v)-\lambda(v)\right)$. Let $\mathcal{O}$ be an orientation of $G$ such that $f(\mathcal{O})$ is minimal. If $f(\mathcal{O})=0$ then any vertex $v$ has indegree bounded by $\lambda(v)$. Assume $f(\mathcal{O})>0$. Let $x_{0}$ be a vertex such that $\mathrm{d}^{-}\left(x_{0}\right)>\lambda\left(x_{0}\right)$ and let $I\left(x_{0}\right)$ be the set of the vertices $v \in V(G)$ such that there exists a directed path (with respect to $\mathcal{O}$ ) from $v$ to $x_{0}$. As no vertex in $I\left(x_{0}\right)$ has an arc coming from the outside of $I\left(x_{0}\right)$, we get $\left|E\left(G\left[I\left(x_{0}\right)\right]\right)\right|=\sum_{v \in I\left(x_{0}\right)} \mathrm{d}^{-}(v)$. As $\left|E\left(G\left[I\left(x_{0}\right)\right]\right)\right| \leq \sum_{v \in I\left(x_{0}\right)} \lambda(v)$ (according to (10)) and as $I\left(x_{0}\right)$ includes at least a vertex $v$ such that $\mathrm{d}^{-}(v)>\lambda(v)$ (namely $x_{0}$ ) we deduce that $I\left(x_{0}\right)$ also includes a vertex $y$ such that $\mathrm{d}^{-}(y)<\lambda(y)$. By construction there exists a directed path from $y$ to $x_{0}$. By reversing the orientation of the edges of this directed path we get a new orientation $\mathcal{O}^{\prime}$ for which all the indegrees but those of $y$ and $x_{0}$ remain the same, the indegree of $y$ increases by one and the indegree of $x_{0}$ decreases by one. Thus $f\left(\mathcal{O}^{\prime}\right)<f(\mathcal{O})$, a contradiction.

Given a 2-connected bipartite plane graph $G=\left(V_{\circ}, V_{\bullet}, E(G)\right)$ with girth 6 whose faces have length 6 , we define:

- the bipartite plane graph $G^{+}=\left(V_{\circ} \cup\{r\}, V_{\bullet}, E\left(G^{+}\right)\right)$obtained from $G$ by adding a vertex $r$ in the outer face linked to the black vertices of this face,

- the bipartite plane multigraph $G_{\|}^{+}=\left(V_{\circ} \cup\{r\}, V_{\bullet}, E\left(G_{\|}^{+}\right)\right)$obtained from $G^{+}$by doubling every edge.

Lemma 3. The graph $G_{\|}^{+}$has an orientation $\mathcal{O}_{\|}^{+}$such that every vertex in $V_{\circ} \cup V$. has indegree 3 and $r$ is a source.

Proof. For every $A \subseteq V_{\circ} \cup V_{\bullet}$ we have $2\left|E\left(G^{+}\right)[A]\right| \leq 3|A|-6$, according to Euler's formula. Hence $\left|E\left(G_{\|}^{+}\right)[A]\right| \leq 3|A|-6$. Let $\lambda$ is the mapping from $V\left(G_{\|}^{+}\right)$to $\mathbb{N}$ defined by $\lambda(v)=0$ if $v=r$, and $\lambda(v)=3$ otherwise. Then, for any $A \subseteq V\left(G_{\|}^{+}\right)$, we have $\left|E\left(G_{\|}^{+}\right)[A]\right| \leq \sum_{v \in A} \lambda(v)$. As $\left|E\left(G_{\|}^{+}\right)\right|=\sum_{v \in V\left(G_{\|}^{+}\right)} \lambda(v)$, it follows from Lemma 2 that $G_{\|}^{+}$has an orientation $\mathcal{O}_{\|}^{+}$such that $d^{-}(v)=\lambda(v)$ for every vertex.

Given such an orientation $\mathcal{O}_{\|}^{+}$of $G_{\|}^{+}$, we define:

$-\Upsilon\left(\mathcal{O}_{\|}^{+}\right)$is the set of the edges $\{x, y\}$ of $G^{+}$such that both $(x, y)$ and $(y, x)$ are arcs of $G_{\|}^{+}$, 
- $\Omega\left(\mathcal{O}_{\|}^{+}\right)$is the orientation of $G^{+}$such that an edge $\{x, y\}$ is oriented from $x$ to $y$ if either both arcs linking $x$ and $y$ in $G_{\|}^{+}$are oriented from $x$ to $y$ with respect to $\mathcal{O}_{\|}^{+}$, or $\{x, y\} \in \Upsilon\left(\mathcal{O}_{\|}^{+}\right)$and $y$ is white.

- the type of a vertex $v$ as 1 if either $v$ has two incoming edges coming from one of its neighbors, or as 2 if the three incoming edges of $v$ come from different neighbors of $v$.

Fact 1. Assume every vertex in $V_{\circ} \cup V_{\bullet}$ is of type 1 in $G_{\|}^{+}$with respect to $\mathcal{O}_{\|}^{+}$. Then:

- The vertex $r$ is a source of $G^{+}$with respect to $\Omega\left(\mathcal{O}_{\|}^{+}\right)$,

- the set $\Upsilon\left(\mathcal{O}_{\|}^{+}\right)$is a perfect matching of $G$,

- each vertex $v \in V$ • has exactly one incoming edge in $G^{+}$, and this edge does not belong to $M$,

- each vertex $v \in V_{\circ}$ has exactly two incoming edge in $G^{+}$, exactly one of which belongs to $M$.

Proof. The vertex $r$ is a source of $\Omega\left(\mathcal{O}_{\|}^{+}\right)$by construction, as it is a source of $\mathcal{O}_{\|}^{+}$. Also, no edge incident to $r$ in $G^{+}$belongs to $\Upsilon\left(\mathcal{O}_{\|}^{+}\right)$. Every vertex different from $r$ has indegree 1 in $G^{+} \backslash \Upsilon\left(\mathcal{O}_{\|}^{+}\right)$with respect to $\Omega\left(\mathcal{O}_{\|}^{+}\right)$as it is of type 1 in $G_{\|}^{+}$. The set $\Upsilon\left(\mathcal{O}_{\|}^{+}\right)$is a perfect matching of $G$ as it is obviously a 1 -factor (each vertex of $G=G^{+}-r$ has degree 1 in $\Upsilon\left(\mathcal{O}_{\|}^{+}\right)$). The last two items also follows directly from the definition of $\Omega\left(\mathcal{O}_{\|}^{+}\right)$.

Lemma 4. Let $\mathcal{O}_{\|}^{+}$be an orientation of $G_{\|}^{+}$such that $r$ is a source and every vertex in $V_{\circ} \cup V_{\bullet}$ has indegree 3 and type 1 . Consider the orientation $\Omega\left(\mathcal{O}_{\|}^{+}\right)$ of $G^{+}$. Each disk $D$ of $G$ is such that $\left|\omega^{-}(D)\right| \geq 3$.

Proof. Let $M=\Upsilon\left(\mathcal{O}_{\|}^{+}\right)$, let $\gamma$ be the outer face of $G^{+}[D]$ and $2 l$ its length, let $\omega_{\circ}\left(\right.$ resp. $\left.\omega_{\bullet}\right)$ be the subset of $\omega(D)$ formed by the edges having an endpoint in $D \cap V_{\circ}$ (resp. $\left.D \cap V_{\bullet}\right)$.

The summation of the indegrees of the vertices in the subgraph $G_{\|}^{+}[D]$ gives: $2\left|E\left(G^{+}[D]\right)\right|=3|D|-2\left|\omega^{-}(D) \backslash M\right|-|\omega(D) \cap M|$. The value $2\left|E\left(G^{+}[D]\right)\right|$ is also the sum of the length of the faces of $H$ thus, as all the interior faces have length 6 and as the outer face as length $2 l$, we get $2\left|E\left(G^{+}[D]\right)\right|=6\left(\left|E\left(G^{+}[D]\right)\right|-\right.$ $|D|+1)+2 l$, that is: $2\left|E\left(G^{+}[D]\right)\right|=3|D|-l-3$. Hence $3|D|-2\left|\omega^{-}(D) \backslash M\right|-$ $|\omega(D) \cap M|=3|A|-l-3$. As $\left|\omega^{-}(D)\right|=\left|\omega^{-}(D) \backslash M\right|+|\omega \circ \cap M|$, we deduce

$$
\left|\omega^{-}(D)\right|=l+3-\left(\left|\omega^{-}(D) \backslash M\right|+\left|\omega_{\bullet} \cap M\right|\right)
$$

Let $t$ (resp. $z$ ) denotes the number of white vertices of $\gamma$ which are matched in $\gamma$ and is a sink of $\gamma$ (resp. not a sink of $\gamma$ ). Obviously $t+z=|\gamma \cap M|$. Every sink of $\gamma$ has indegree 2 in $\gamma$ hence is a white vertex of $\gamma$ and is matched in $\gamma$. It follows that $t$ is the number of sinks of $\gamma$ hence also the number of sources of 
$\gamma$. Let $e \in \omega^{-} \backslash M$. Then either $e$ is incident to a source of $\gamma$, or it is incident to a white vertex which is matched in $\gamma$ and which is not a sink of $\gamma$. It follows that $\left|\omega^{-}(D) \backslash M\right| \leq t+z=|\gamma \cap M|$. Also, $|\gamma \cap M|+\left|\omega_{\bullet} \cap M\right| \leq l$ as this is the number of black vertices of $\gamma$ matched in $\omega \bullet \cup \gamma$. Altogether, we get $\left|\omega^{-}(D) \cap M\right|+\left|\omega_{\bullet} \cap M\right| \leq|\gamma \cap M|+\left|\omega_{\bullet} \cap M\right| \leq l$. Thus $\left|\omega^{-}(D)\right| \geq 3$, according to (2).

Definition 1. Le $v$ be a vertex of type 2 , and let $x_{1}, y_{1}^{1}, \ldots, y_{1}^{a_{1}}, x_{2}, y_{2}^{1}, \ldots, y_{2}^{a_{2}}$, $x_{3}, y_{3}^{1}, \ldots, y_{3}^{a_{3}}$ be the neighbors of $v$ in circular order, where $x_{1}, x_{2}, x_{3}$ are the three neighbors of $v$ incident to an arc oriented to $v$. The splitting of $v$ is obtained by replacing $v$ by three vertices $v_{1}, v_{2}, v_{3}$ and dispatching the arcs incident to $v$ to $v_{1}, v_{2}, v_{3}$ : for $i \in\{1,2,3\}$, the arcs incident to $v_{i}$ are: one arc to $x_{i}$, one arc from $x_{i}$, for each $j=1, \ldots, a_{1}$ two arcs to $y_{i}^{j}$, and two arcs coming from $x_{i+1}$ (if $i<3$, or $x_{1}$ if $i=3$ ).

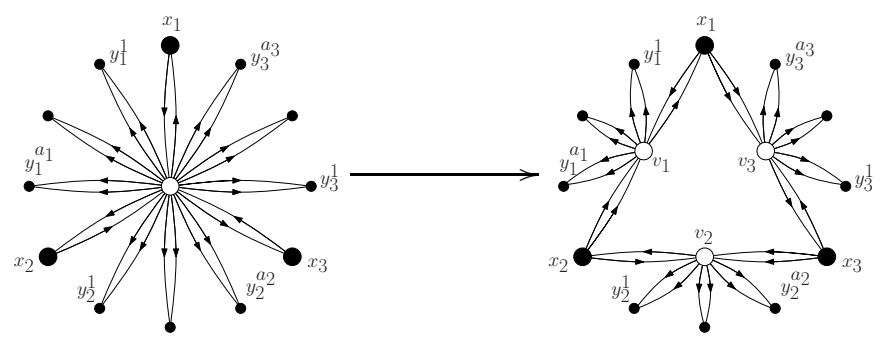

Remark that the splitting of a vertex $v$ of type 2 into $v_{1}, v_{2}, v_{3}$ preserves the length of the faces and the indegrees. After the splitting, each of $v_{1}, v_{2}, v_{3}$ is of type 1 .

Lemma 5. Let $\mathcal{O}_{\|}^{+}$be an orientation of $G_{\|}^{+}$such that $r$ is a source and every vertex in $V_{\circ} \cup V_{\bullet}$. has indegree 3 and such that every vertex in $V_{\circ}$ has type 1 . Consider the orientation $\Omega\left(\mathcal{O}_{\|}^{+}\right)$of $G^{+}$. Each disk $D$ of $G$ is such that $\sigma(D) \geq 3$.

Proof. We proceed by induction on the number of vertices in $V$. which have type 2 . If no vertex in $V \bullet$ has type 2 , the result follows from Lemma 4 Assume that the result holds if $V$. includes at most $k$ vertices of type $2(k \geq 0)$ and assume $V$ • includes $k+1$ vertices of type 2 . Let $v$ be one of them. Let $G^{\prime}$ be the multigraph obtained from $G_{\|}^{+}$by splitting $v$ into three vertices $v_{1}, v_{2}, v_{3}$. Let $H$ be the unique plane graph such that $H_{\|}^{+}=G^{\prime}$. Let $F$ be the circuit of length 6 including $v_{1}, v_{2}, v_{3}$ arising in $H^{+}$from the splitting of $v$. Let $D$ be a disk of $G$. If $v \notin \operatorname{Extr}(D)$ then the result follows from the induction applied to the graph $H_{\|}^{+}$obtained from $G_{\|}^{+}$by splitting $v$ (the $\sigma$-value of $D$ in $G^{+}$will be the same has the $\sigma$-value of $\operatorname{Clos}(\operatorname{Extr}(D))$ in $\left.H^{+}\right)$. Otherwise, let $D^{\prime}$ be the disk of $H^{+}$ obtained from $D-v$ by adding those of $v_{1}, v_{2}, v_{3}$ having at least two neighbors in $D-v$. Then the set of edges $\omega^{-}\left(D^{\prime}\right)$ (in $H^{+}$) is the union of the set $\omega^{-}(D)$ (computed in $G^{+}$) and of the set of edges in $\omega^{-}\left(D^{\prime}\right) \cap F$. As the outer face of $H^{+}\left[D^{\prime}\right]$ meets $F$ on an interval (possibly reduced to a vertex) and as $F$ is a circuit, $\left|\omega^{-}\left(D^{\prime}\right) \cap F\right| \leq 1$. As $v$ was a source of $G^{+}$and none of $v_{1}, v_{2}, v_{3}$ are, we 
get that the $\sigma$-value of $D$ in $G^{+}$is at least equal to the $\sigma$-value of $D^{\prime}$ in $H^{+}$, which in turn is at least 3 by induction. Hence $\sigma(D) \geq 3$, what completes the proof of the induction.

Lemma 6. Let $\mathcal{O}_{\|}^{+}$be an orientation of $G_{\|}^{+}$such that $r$ is a source and every vertex in $V_{\circ} \cup V_{\bullet}$. has indegree 3 and such that every vertex in $V_{\circ}$ has type 1 . Consider the orientation $\Omega\left(\mathcal{O}_{\|}^{+}\right)$of $G^{+}$and its restriction to $G$. Then $G$ is the incidence graph of a contact system of segments and points with the embedding and the orientation induced by the contact system.

Proof. This is a direct consequence of Lemma 5 and Lemma 1

Consider a contact family $\mathcal{F}$ of triangles and segments. Each triangle is formed by three segments. The contact family thus defines a bipartite incidence graph of segments and points, in which the segments of $\mathcal{F}$ appear as vertices, and the triangles of $\mathcal{F}$ appear as faces of length 6 .
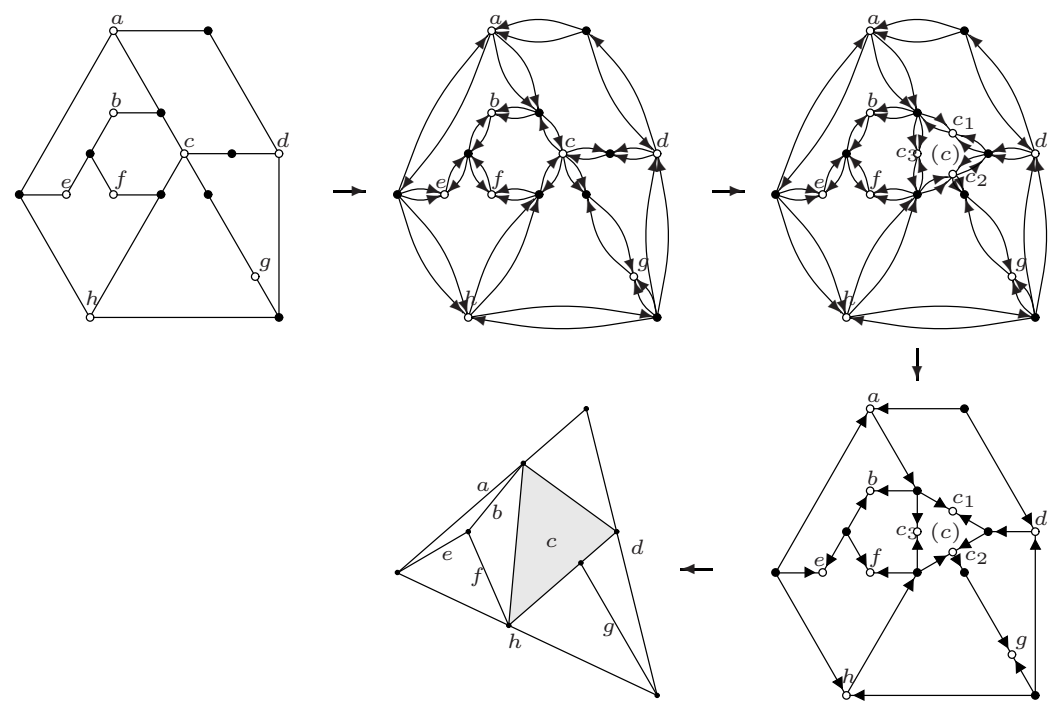

Fig. 1. Representation of a planar linear hypergraph by contacts of triangles and segments. First figure is the representative graph $B$ of the hypergraph, second figure is the orientation of $B_{\|}^{+}-r$, third figure is the orientation of $\Gamma_{\|}^{+}-r$ obtained from $B_{\|}^{+}-r$ by splitting of vertices of type 2 (here $c$ is split into $c_{1}, c_{2}, c_{3}$ ), fourth figure is the associated orientation of $\Gamma$. The last figure is the deduced representation of the hypergraph.

Conversely, to represent planar linear hypergraph as the incidence graph of a family of triangles and segments in contacts, we will derive from the representative plane graph $B$ of the hypergraph a bipartite plane graph $\Gamma$, so that each vertex of $B$ will correspond either to a vertex or a face of length 6 of $\Gamma$, 
and deduce the representation of $B$ from a representation of $\Gamma$ as the incidence graph of a family of segments and points (see Fig. 1] and 2).

Although the exact details of the construction of an actual family of segments and points whose incidence graph is $\Gamma$ can be found in [8], we shall give some intuition of how it works: By using augmentations one reduces to the case where $\Gamma$ have exactly 3 sources lying on the outer face. Then these vertices are embedded into 3 points of the plane in general position forming a triangle $T_{0}$. The orientation and plane embedding of the bipartite graph are translated into a linear system which resolution gives the embedding of the remaining black vertices in the interior of $T_{0}$. In this embedding, the white vertices correspond to the straight line segments which endpoints will be the points corresponding to the 2 incoming black vertices. This construction is described in 8]. A hint of these two steps is given in the last two drawings of Fig. 1. According to the construction of $\Gamma$, a representation of the hypergraph $\mathcal{H}$ by contacts of triangles and segments follows.

Proof of the Main Theorem. Let $\mathcal{H}$ be a planar linear hypergraph. We may assume without loss of generality that $\mathcal{H}$ has order and size at least 3 . We may also assume that the representative plane graph $B=\left(V_{\circ}, V_{\bullet}, E(B)\right)$ of $\mathcal{H}$ is 2-connected and that every face of $B$ have length 6 , as this may be achieved by adding some dummy vertices to $\mathcal{H}$ without creating any additional adjacencies between the original vertices of $\mathcal{H}$. A representation of $\mathcal{H}$ by contact of triangles is then deduced from a representation of the augmented hypergraph by deleting the triangles corresponding to the dummy vertices:
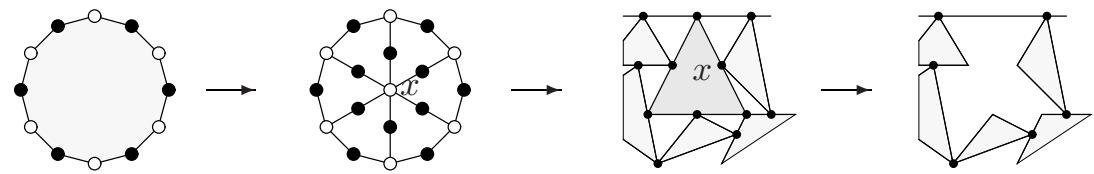

According to Lemma 3. $B_{\|}^{+}$has an orientation such that every vertex in $V_{\circ} \cup V_{\bullet}$ has indegree 3 and $r$ is a source. By splitting all the vertices in $V_{\text {。 }}$ having type 2 , we get a graph $\Gamma_{\|}^{+}$(associated with a bipartite plane graph $\Gamma$ ) and an associated orientation of $\Gamma^{+}$, so that each vertex of $B$ corresponds either to a vertex or a face of length 6 of $\Gamma$ whose edges are oriented from the black vertices. According to Lemma 6, the graph $\Gamma$ is the incidence graph of a contact system of segments and points with the embedding and the orientation induced by the contact system. As the faces of length 6 coming from splittings are then empty triangles, we deduce a representation of $\mathcal{H}$.

\section{Extensions and Open Problems}

The incidence poset of a graph (or more generally of a hypergraph) is the poset where the only covers are defined by $x<e$ if $x$ is a vertex, $e$ is an edge and $e$ is incident to $x$. The dimension $\operatorname{dim} \mathbf{P}$ of $\mathbf{P}=(X, P)$ is the least positive integer 


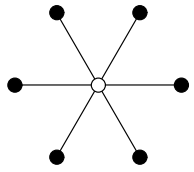

1

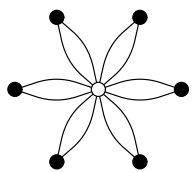

Type 1
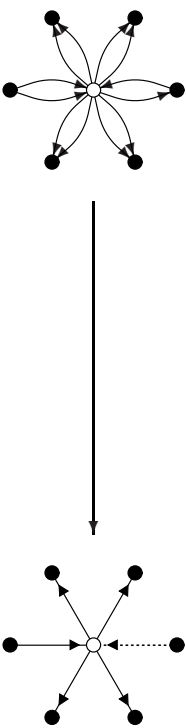

$\downarrow$
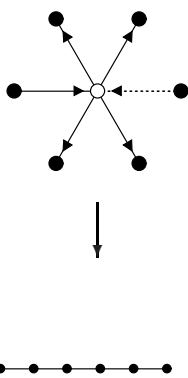
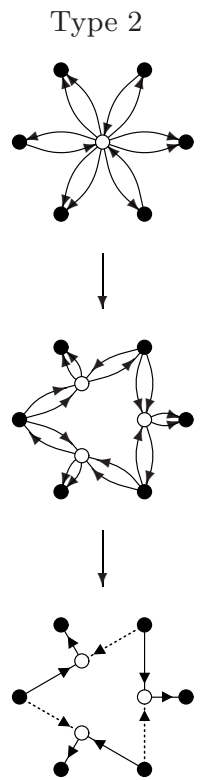

1

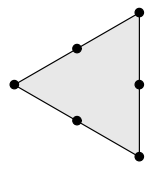

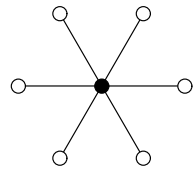

$\downarrow$

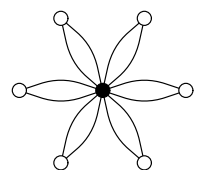

Type 1

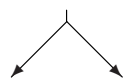

Type 2
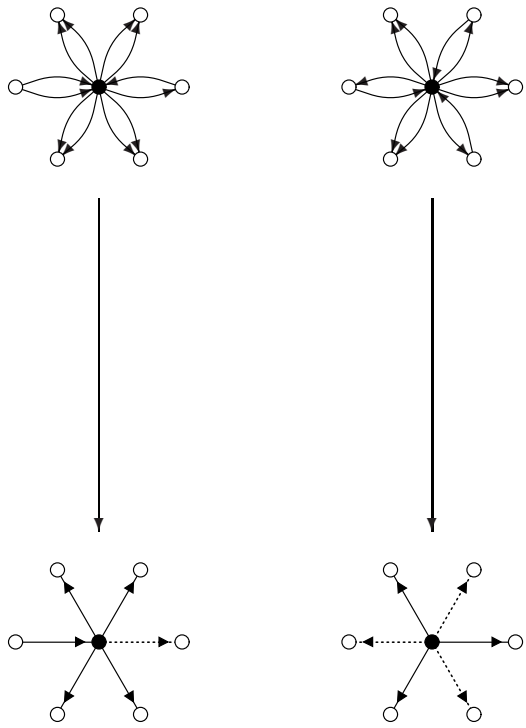

1

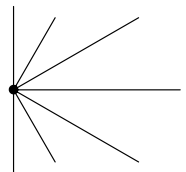

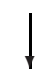

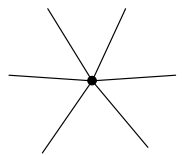

Fig. 2. From the representative graph to the contact representation. Neighborhoods of vertices are shown in $B$ (first row), in $B_{\|}^{+}$(second row), in $B_{\|}^{+}$with orientation $\mathcal{O}_{\|}^{+}$ (third row), in $\Gamma_{\|}^{+}$with orientation $\mathcal{O}_{\|}^{+}$(fourth row), in $\Gamma^{+}$with orientation $\mathcal{O}^{+}$where dashed edges representing $M$ (fifth row), in the contact representation (sixth row). 
$t$ for which there exists a family $\mathcal{R}=\left(<_{1},<_{2}, \ldots,<_{t}\right)$ of linear extensions of $P$ so that $P=\bigcap \mathcal{R}=\bigcap_{i=1}^{t}<_{i}$. This concept has been introduced by Dushnik and Miller in [6]. A family $\mathcal{R}=\left(<_{1},<_{2}, \ldots,<_{t}\right)$ of linear orders on $X$ is called a realizer of $P$ on $X$ if $P=\bigcap \mathcal{R}$. For an extended study of partially ordered sets, we refer the reader to 25 .

A celebrated theorem of Schnyder states that a graph is planar if and only if its incidence poset has dimension at most 3 [22]. Although the incidence poset of a simple planar hypergraph $\mathcal{H}$ has dimension at most 3 (what follows from [3]), the converse is false: The linear hypergraph $\mathcal{H}$ with vertices $1, \ldots, 5$ and edge set $\{\{1,2\},\{2,3\},\{3,4\},\{1,4\},\{1,3,5\},\{2,4,5\}\}$. is not planar $(\mathrm{B}(\mathcal{H})$ is a subdivision of $\left.K_{3,3}\right)$ though its incidence poset has a realizen 2 of size 3 .

Schnyder's theorem is generalized in [17] to a sufficient condition for the geometric realizability of an abstract simplicial complex and in 18 to a general representation theorem for posets. From this later theorem, it is easily deduced that the vertices of simple hypergraphs with incidence posets of dimensions $d$ can be represented by convex sets of the Euclidean space of dimension $d-1$, in such a way that the edges of the hypergraph are exactly the maximal subsets of vertices, such that the corresponding subset of convexes has a non-empty intersection. Thus, in some way, hypergraphs with incidence poset of dimension 3, although not necessarily planar, still have a strong relation with the plane. This encourages the following conjecture:

Conjecture 2. Any linear hypergraph with incidence poset of dimension at most 3 is the intersection hypergraph of a family of triangles and segments in the plane.

\section{References}

1. Andreev, E.M.: On convex polyhedra in Lobačevskiǐ spaces. Matematicheskii Sbornik 81, 445-478 (1970)

2. Berge, C.: Graphes et hypergraphes, 2nd edn. Dunod, Paris (1973)

3. Brightwell, G., Trotter, W.T.: The order dimension of planar maps. SIAM journal on Discrete Mathematics 10(4), 515-528 (1997)

4. Cori, R.: Un code pour les graphes planaires et ses applications, Société Mathématique de France, Paris, vol. 27 (1975)

5. Cori, R., Machì, A.: Maps, hypermaps and their automorphisms. Expo. Math. 10, 403-467 (1992)

6. Dushnik, B., Miller, E.W.: Partially ordered sets. Amer. J. Math. 63, 600-610 (1941)

7. de Fraysseix, H., Ossona de Mendez, P.: Intersection Graphs of Jordan Arcs, Contemporary Trends in Discrete Mathematics. In: DIMATIA-DIMACS. DIMACS Series in Discrete Mathematics and Theoretical Computer Science, Štiřin 1997 Proc., pp. 11-28 (1999)

8. de Fraysseix, H., Ossona de Mendez, P.: Barycentric systems and stretchability. Discrete Applied Mathematics 155(9), 1079-1095 (2007)

\footnotetext{
$<_{1}: \quad 2<1<\{1,2\}<3<\{2,3\}<5<\{1,3,5\}<4<\{2,4,5\}<\{1,4\}<\{3,4\}$

2 Take $<2: \quad 5<4<2<2<\{2,4,5\}<3<3<\{3,4\}<\{2,3\}<1<\{1,3,5\}<\{1,2\}<\{1,4\}$. $<3: \quad 1<4<\{1,4\}<3<\{3,4\}<5<\{1,3,5\}<2<\{2,4,5\}<\{1,2\}<\{2,3\}$
} 
9. de Fraysseix, H., Ossona de Mendez, P.: On representations by contact and intersection of segments. Algorithmica 47(4), 453-463 (2007)

10. de Fraysseix, H., Ossona de Mendez, P., Rosenstiehl, P.: On triangle contact graphs. Combinatorics, Probability and Computing 3, 233-246 (1994)

11. de Fraysseix, H., Pach, J., Pollack, R.: Small sets supporting Fary embeddings of planar graphs. In: 20th Annual ACM Symposium on Theory of Computing, pp. 426-433 (1988)

12. de Fraysseix, H., Pach, J., Pollack, R.: How to draw a planar graph on a grid. Combinatorica 10, 41-51 (1990)

13. Johnson, D.S., Pollak, H.O.: Hypergraph planarity and the complexity of drawing Venn diagrams. Journal of Graph Theory 11(3), 309-325 (1987)

14. Jones, R.P.: Colourings of hypergraphs, Ph.D. thesis, Royal Holloway College, Egham, p. 209 (1976)

15. Koebe, P.: Kontaktprobleme der konformen Abbildung. Ber. Verh. Schs. Akad. Wiss. Leipzig, Math.-Phys. Kl. 88, 141-164 (1936)

16. Ossona de Mendez, P.: Orientations bipolaires, Ph.D. thesis, Ecole des Hautes Etudes en Sciences Sociales, Paris (1994)

17. Ossona de Mendez, P.: Geometric Realization of Simplicial Complexes, Graph Drawing. In: Kratochvíl, J. (ed.) GD 1999. LNCS, vol. 1731, pp. 323-332. Springer, Heidelberg (1999)

18. Ossona de Mendez, P.: Realization of posets. Journal of Graph Algorithms and Applications 6(1), 149-153 (2002)

19. Rosenstiehl, P., Tarjan, R.E.: Rectilinear planar layout and bipolar orientation of planar graphs. Discrete and Computational Geometry 1, 343-353 (1986)

20. Scheinerman, E.R.: Intersection classes and multiple intersection parameters of graphs, Ph.D. thesis, Princeton University (1984)

21. Scheinerman, E.R., West, D.B.: The interval number of a planar graph: Three intervals suffice. Journal of Combinatorial Theory, Series B 35, 224-239 (1983)

22. Schnyder, W.: Planar graphs and poset dimension. Order 5, 323-343 (1989)

23. Schnyder, W.: Embedding planar graphs in the grid. In: First ACM-SIAM Symposium on Discrete Algorithms, pp. 138-147 (1990)

24. Tamassia, R., Tollis, I.G.: Tessalation representation of planar graphs. In: Proc. Twenty-Seventh Annual Allerton Conference on Communication, Control, and Computing, pp. 48-57 (1989)

25. Trotter, W.T.: Combinatorics and partially ordered sets: Dimension theory. John Hopkins series in the mathematical sciences. Johns Hopkins University Press, London (1992)

26. Walsh, T.R.S.: Hypermaps versus bipartite maps. J. Combinatorial Theory 18(B), 155-163 (1975)

27. White, A.T.: Graphs, Groups and Surfaces, revised edn. Mathematics Studies, vol. 8. North-Holland, Amsterdam (1984)

28. Zykov, A.A.: Hypergraphs. Uspeki Mat. Nauk 6, 89-154 (1974) 